\title{
TRES MODELOS EDUCATIVOS PRESENTES EN LAS SOCIEDADES ACTUALES YLOS SISTEMAS EVALUATIVOS
}

\author{
Mg. Alessandro Caviglia Marconi
}

\section{RESUMEN}

El presente artículo tiene como objetivo presentar tres modelos educativos que se encuentran en competencia en las sociedades actuales, para sacar a luz los criterios evaluativos presentes en cada uno. El autor señala que los tres modelos en cuestión son a) el modelo aristocrático, b) el modelo neoliberal $y \mathrm{c}$ ) el modelo democrático. El modelo aristocrático está hecho a la medida de los intereses de las élites aristocráticas que regentan muchas sociedades $y$ tienen como punta de lanza el eslogan de una "educación en valores" privados. El modela neoliberal es fomentado por las élites económicas $y$ tiene como eje central perpetuar el sistema económico $y$ hacer de los educandos personas funci..,nales al mercado. Se trata de una educación para el lucro que entiende la educación como un negocio. Finalmente, el modelo democrático tiene como objetivo el formar ciudadanos, dotados de capacidad reflexiva $y$ crítica, para lo cual es importante la formación integral de la persona, añadiendo a la formación científica y técnica la formación en las humanidades $y$ artes. Se presenta cuáles son los criterios evaluativos de cada modelo y cómo, peligrosamente para las sociedades democráticas $y$ la libertad de los ciudadanos, el modelo democrático se encuentra jaqueado por la fuerza que han adquirido los otros dos modelos.

\begin{abstract}
This article presents three educational models which are in competence in current societies, to show evaluative criteria of every one. The author indicates that the three models are: a) Aristocratic model, b) Neo Liberal model and c) Democratic model. The Aristocratic model is developed according to aristocratic elites which manage many societies and has a slogan of prívate "Values-based Education". The Neo liberal model is managed by economic elites and has the mission to perpetuate the economic system and made of the learners functional persons to the market. It is an education far profit which conceives itas a bus ine ss. Finally the Democratic model has the objective to train citizens with reflexive and critica! attitude far what it is important a whole education of the person with scientific and technical aspects and humanities and arts. Evaluative criteria far every model are presented and the danger in which democratic societies and free citizens, the Democratic model is checked bythe force ofthe othertwo mode ls .
\end{abstract}

\section{PALABRAS CLAVE}

Educación, modelo educativo, evaluación, democracia, sociedad.

\section{KEYWORDS}

Education, educa tio n, model, evaluation, democracy, society. 
$\mathrm{E}$ scribi r sobre la evaluación educativa supone aclarar un conjunto de presupuestos. Al ento de evaluar sí la educación ha alcanzado sus objetivos, es necesario preguntarse previamente cuáles son dichos objetivos. Puesto que la actividad educativa se realiza en sociedades concretas, dichos objetivos y parámetros evaluativos dela educación tiene que ver con las exigencias ele las sociedades en cuestión. Como toda institución, las instituciones educativas, las escuelas, los institutos técnicos y las universidades, se encuentran insertas en sociedades concretas. En tal sentido, al interior ele tales instituciones, la educación se presenta como una práctica social que tiene como finalidad forjar personas en adultos y ciudadanos ${ }^{1}$. En el mundo contemporáneo, la educación se presenta corno una cuestión de suma irnportancia, debido a que ele ella depende el tipo ele sociedad que queramos gestar. En este sentido, tres modelos diferentes se disputan la conducción de la educación en el Perú y en el mundo actual: a) el modelo aristocrático, b) el modelo neoliberal, y c) el modelo democrático.

En el Perú y en el mundo contemporáneo asistimos a la competencia entre estas tres tendencias en la educación. lo característico de ellas es que todas expresan proyectos políticos diferentes. Este elemento no es algo negativo per sé,pues no se trata de tergiversar la práctica de la educación sometiéndola a ambiciones políticas partidarias, sino que nos hallamos ante modos de pensar la educación considerando que ella debe establecer una conexión con lo que queremos como sociedad. Esto es así, porque la educación es una actividad socíal2 y las instituciones educativas se encuentran insertas en sociedades concretas ante las cuales tiene una doble actitud: de una parte, dan respuesta a exigencias planteadas por el entorno social, mientras que de otras ejercen un papel de crítica de las actitudes y creencias no reflexionadas que subsisten en la sociedad.

Agentes políticos diferentes se encuentran comprometidos con alguno ele los modelos señalados. Pero, además, podemos encontrar que algunos de ellos tienen puntos de confluencias que permiten que se potencien mutuamente. Una cuestión preocupante resulta ser el que en el juego de fuerzas políticas institucionales el modelo democrático está perdiendo el terreno que tenía en países importantes como los Estados Unidos, los países europeos y la India. En el Perú el modelo democrático nunca ha tenido el arraigo deseado. La escuela, la universidad y los teóricos más influyentes han apostado más por una educación autoritaria y aristocrática, que por una de carácter dialogante y democrática, y actualmente el modelo neoliberal tiene una preocupante penetración. Esto también tiene que ver con el hecho de que el debate y la discusión sobre la educación han sido desarticulados durante los últimos años, a pesar de que hay intelectuales, pedagogos y experiencias educativas sumamente importantes y sugerentes.

La influencia del pensamiento antídemocrático en el mundo contemporáneo es preocupante por dos razones fundamentales. La primera es que la democracia, más que ser un modelo político que sirva para organizar una sociedad, es en nuestros días un principio ético fundamental que tiene como objeto evaluar los sistemas políticos y sociales; en este sentido, la democracia es formar parte dela ética dela política, como lo constituyen también elementos como la defensa de los derechos fundamentales o el cumplimiento ele los derechos humanos ${ }^{3}$. La segunda razón es que la democracia permite formar ele mejor manera a las personas, porque permite que ellas aprendan a hacer un uso razonable de su libertad y puedan convertirse en ciudadanos cooperantes en la sociedad a fin de enfrentar de manera más eficazlos problemas. En este sentido, es conocida la afirmación del filósofo y pedagogo estadounidense John Dewey, según la cual la democracia permite el usosocial delainteligencia.

En lo que sigue, presentaré brevemente los tres modelos educativos (1) para, seguidamente, presentar los parámetros evaluativos que cada uno de ellos plantea, realizando un examen crítico de los mismos (2). Finalmente, señalaré las conclusiones a las que me conducen estas reflexiones (3).

\section{l. Los tres modelos educativos}

Los modelos educativos que recorren las sociedades contemporáneas se encuentran impulsados por instituciones y agenrés políticamente orientados. Hace unas décadas el modelo democrático tenía mayor apoyo de instituciones, pero en la actualidad está siendo lamentablemente relegado y tanto el modelo aristocrático como el neolíberal están volviéndose cada vez más dominant es. Pero el hecho de que estos modelos hayan devenido en dominantes no tiene relación con la calidad de sus proyectos educativos, sino porque las instituciones y personas que los impulsan han adquirido poder político, simbólico y económico, además por el auge que tiene el pensamiento político antidemocrátíco a lo largo del planeta. La reflexión crítica permitirá discernir 
entre el poder de un modelo dominante y su corrección. De otra parte, dichos modelos pueden confluir en la consecución de ciertos objetivos, y las instituciones y los agentes que se encuentran motivados por ellos pueden llegar a alianzas estratégicas, dependiendo del poder que tales instituciones y personas tengan en las sociedades.

\section{$1.1 \quad$ Elmodelo aristocrático}

los grupos más conservadores de las sociedades modernas fomentan el modelo aristocrático en la educación. los representantes máf influyentes de estos grupos son de carácter religiosoy presentan una educación que se enfrenta a enemigrn\muy concretos: el materialismo y el ateísmo. De e a manera, los defensores de este modelo afirman la s\lpremacía de un a educación espiritualista.

Dicho carácter espiritualista se encuentra acompañado de una perspectiva axiomática y exclusivista de lo que ellos denominan "valores". El slogan principal de este modelo es el de la "educación en valores". Dicha educación supone la existencia de un listado único, universal e invariable de valores que u1 , lpersona debe incorporar en su vida para tener una formación sólida. Pero la idea de este elenco viene acompañada ele la creencia de que tales valores tienen una naturaleza axiomática, es decir, los valores son axiomas que no se pueden demostrar, sino que sólo se deben aceptar como puntos de partida de nuestras acciones y orientaciones en la vida. Son los principios y fines últimos incuestionables que deben guiar una vida "auténticamente humana". Esto es así, porque tales principios están conectados con una supuesta Verdad trascendente y misteriosa, a la que sólo algunos ungidos pueden acceder por medio de poderes divinos y que son inaccesibles a través del razonamiento.

Una variante del modelo aristocrático de educación supone que los valores en cuestión son hallados por el ser humano en h naturaleza, siempre que -según se argumenta- los seres humanos hagan un uso adecuado de sus facultades racionales. En este contexto "hacer un uso adecuado de la razón" significa no salir de los parámetros axiológicos, por más que ello signifique una limitación al uso libre de la capacidad de razonar. Esto supone afirmar dos tesis fuertes respecto de los valores y la moral: la primera es la objetividad léxica de los valores; la segunda, es la del realismo ontológico de los mismos. la tesis de la objetividad de los valores supone que éstos existen independientemente de las creencias de las personas y que nuestro lenguaje moral refiere a entidades que se encuentran allí fuera en el mundo, mientras que el realismo de los valores sostiene que éstos conforman la estructura esencial de la realidad. En el debate ético contemporáneo se ha argumentado, de manera sólida, en contra de ambas tesis ${ }^{4}$.

Planteados de esta manera, los valores se presentan como exigencias que se sustraen al escrutinio razonado y libre de las personas. Se trata de un conjunto de principios, de naturaleza axiomática, que son impermeables al cuestionamiento, la crítica, al razonamiento y a la justificación. Quien se atreva a cuestionarlos es sindicado inmoral y perverso, se lo considera una persona sospechosa, pues no cree en los valores "que toda persona decente" debe asumir". Por otra parte, los valores vistos de esta manera son ele carácter moral, únicos y rivalizan con otras listas de valores que personas pertenecientes a culturas subordinadas mantienen.

En este contexto, educar en valores significa obligar a los estudiantes a que abandonen sus propias listas de valores para que acepten la lista que las instituciones educativas quieren imponer corno dominantes. De este modo, "educar" es una versión sofisticada de la antigua práctica católica de extirpar idolatrías y extinguir herejías. Pero, además, consiste en perpetuar el poder de la élite aristocrática dominante. El objetivo de esta élite es crear en las personas una identidad singularista (Ser: Identidad y violencia). Una identidad singularista es aquella que una persona constituye cuando piensa que su identidad se encuentra iluminada por uno solo de los múltiples focos identitarios. Élites aristocráticas o líderes fundamentalistas pueden apagar sistemáticamente los focos que con forman la identidad compleja de las personas y hacerlas creer que sólo son una cosa, aquella cosa que la lista de valores especifica (por ejemplo, le inculcan la idea que antes de todo son creyentes católicos, dejando de lado el hecho de ser hombres o mujeres que se dedican a tal o cual profesión o estudio, y que tiene tales o cuales aficiones). De hecho, esta estrategia es sumamente efectiva ya que goza ele la complicidad silenciosa de los educandos por el hecho de que asumir una identidad compleja es más difícil que aceptar una eliminación de aquella complejidad. Podemos afirmar que educar a personas para que asuman la complejidad y riqueza de su identidad, con sus múltiples pertenencias, es análogo a lo que Nelson Mandela quiso hacer ele Sudáfrica, una sociedad del arcoíris, ele muchos colores y razas conviviendo en un proyecto ele mutuo respeto, colaboración y tolerancia, mientras que forjar una identidad singularista es análogo a la construcción de la sociedad sudafricana marcada por el racisn , y el apart heid ${ }^{5}$. 
Por estas características, el modelo aristocrático es profundamente autoritario y antíclemocrático. Este carácter autoritario se presenta ele múltiples maneras: exigiendo que el razonamiento se allane ante exigencias axiomáticas, y al exigir cierta disciplina a los educando ele un modo que no soporta el examen crítico, como es la obediencia a normas que no soportan el examen racional. Pero, demás, dicho autoritarismo se manifiesta en la manera ele tratar las asignaturas curriculares. Las asignaturas centrales para este modelo las constituyen las ciencias, las asignaturas técnicas, la historia y la filosofía. El resto de las humanidades, como la literatura y las artes son relegadas. Además, el aspecto ele las ciencias valorado es el que concierne al aprendizaje ele teorías científicas, y no se incentiva la creatividad, que constituye un elemento importante para el desarrollo científico.

Entre las ciencias humanas, el modelo aristocrático selecciona la historia y la filosofía, pero de un modo que tergiversa la naturaleza ele dichas disciplinas. En el caso de la historia, en vez ele fomentar la investigación, lo que el modelo privilegia es un relato prefabricado del pasado. Dicho relato se centra en las gestas ele los héroes militares con el objetivo ele generar la imagen ele que todos los pertenecientes a la sociedad forman una unidad carente ele diferencias socioeconómicas, las diferencias religiosas, étnicas, entre otras. Este relato ele la historia da menos importancia a los personajes vinculados a la creación literaria o del pensamiento, pues ello despierta la actitud crítica de los modelos ele vida privilegiados por la educación aristocrática. Un ejemplo claro de la omisión en la enseñanza de la historia se manifiesta en la oposición que el sector tradicionalista peruano ante el intento de incluir en la enseñanza de la historia reciente el proceso ele violencia interna que atravesó el país entre las décadas de los 80 y 90, especialmente la oposición a la inclusión del aporte elela Comisión de la Verdad y Reconciliación ${ }^{6}$.

En el caso ele la enseñanza ele la filosofía, la tendencia autoritaria del modelo ele educación aristocrático se manifiesta en convertir la filosofía en una actividad ele adoctrinamiento en lo que los sectores conservadores denominan "filosofía perenne". Bajo ese rótulo, se enseña a los estudiantes en las escuelas privadas y en las universidades que se encuentran en las manos tales sectores el neotomismo como sí fuese una tradición portadora ele verdades universales e inmutables que deben ser impartidas a fin de combatir lo que denominan el "relativismo" y el "ateísmo" ele las sociedades contemporáneas. Con ello se elimina todo componente reflexivo y crítico a la filosofía, desnaturalizánclola por completo con el fin ele convertirla en una herramienta para sus fines políticos partidarios. Las artes son dejadas completamente ele lado, debido a que ellas fomentan el desarrollo ele la imaginación. La pintura, la música, el teatro y la poesía, por ejemplo, son tomadas como piezas exóticas que tiene como finalidad ornamental, pero ele ninguna manera como elementos importantes del acerbo cultural con el cual deben ser conectados losestudiantes ${ }^{7}$.

La razón por la que orientan la educación en esta dirección es porque buscan el control social de las personas para que acepten un régimen de prerrogativas especiales, donde una élite aristocrática impone las reglas que articulan la sociedad. Los educados bajo dicho modelo aceptan las reglas sociales que imponen la desigualdad ele status social, y la discriminación social, racial y por orientación sexual. De este modo, la educación aristocrática consolida el régimen que Guillermo Nugent denomina "el orden tutelar", que impone el orden propio eleinstituciones jerárquicas, como son las Fuerzas Armadas y las Iglesias a las relaciones entrelas personas al interior elelasociedad 8 .

\subsection{Elmodelo neolíberal}

El modelo neoliberal es planteado por la élite económica, y se presenta como una educación orientada a la ganancia económica y a satisfacer las exigencias del mercado. Se trata de una educación para la ganancia en dos sentidos: en un primer sentido, debido a que las escuelas, los institutos tecnológicos y las universidades se constituyen prioritariamente como un negocio y de un modo marginal se entienden como comunidades educativas; en un segundo sentido, porque se fomenta a los estudiantes estudiar carreras lucrativas.

Los centros educativos, a nivel primario, secundario, terciario y universitario, se constituyen como sociedades con fines de lucro y conciben que su fin prioritario consiste en ganar dinero. Esta lógica ha penetrado las universidades, que originariamente eran instituciones sin fines ele lucro. En muchos países, como es el caso de Chile, las universidades privadas disfrazan el lucro por medio del alquiler de locales, propaganda y otros mecanismos, aunque las leyes locales prohiben que las universidades lucren. En el Perú, gracias al decreto legislativo 882, dado durante el gobierno ele Alberto Fujimori, se permitió la constitución de universidades con fines ele lucro.

\section{1 un1Pé}


Muchas universidades se alinearon a esta normativa, y muchas las que nolo hicieron camuflaron ellucro.

Por otra parte, se incentiva a los estudiantes seguir carreras lucrativas. A través de los medios de comunicación se fomenta la imagen que ser feliz es ser exitoso, y ello significa ganar dinero. Esto se encuentra reforzado por el reiterado discurso del "sujeto empresario", según el cual las personas deben convertirse en empresarios y constituir sus propios negocios porque ello les resultará rentable. Junto con esto, el modelo neoliberal tiene como finalidad insertar a los estudiantes en un carril de carrera lo más temprano posible. Parte ele esta política es eliminar los estudios generales compartidos entre estudiantes ele diferentes carreras en la universidad con el fin de colocarlos directamente en los estudios propios de su disciplina. Ello no permite a los estudiantes ver un conjunto ele materias que enriquezca su formación y tampoco permite el intercambio con estudiantes que tienen otros intereses y visión de las cosas.

la educación para la ganancia tiene como objetivo formar agentes funcionales al mercado, más que ciudadanos ele una sociedad democrática. lo que husca es formar personas que carezcan ele iniciativa y d e conciencia crítica, agentes predecibles, funcionales, generados en serie e intercambiables unos por otros. En este sentido, tiene como objetivo neutralizar o anular la identidad de las personas. Es por esa razón que eliminan las artes y las humanidades, pues ambas generan la capacidad de imaginar mundos distintos y ele tener capacidad crítica. Más bien, lo que privilegia es la enseñanza de la ciencia y la técnica. Pero, al igual que la educación aristocrática, el aspecto ele la ciencia en la que se concentra es en el de la repetición de conocimientos y nola generación de los mismos.

Al igual que la educación aristocrática, esta generación ele individuos funcionales para el mercado y cuya1Llenticlacl ha quedado eclipsada tiene como objetivo el control social de las personas. la penetración del poder del dinero en las instituciones educativas se convierte en una poderosa herramienta de control social, porque las instituciones son entendidas como empresas que se encuentran al servicio delos intereses económicos y políticos delos dueños, los profesores son considerados empleados y los estudiantes son entendidos como clientes. los dueños manejan las instituciones educativas conforme a la lógica de la eficacia que atraviesa todas las esferas del mercado y no con criterios pedagógicos que tengan la finalidad de formar ciudadanos. Al someterse a instituciones educativas marcadas por la lógica de la eficacia y los intereses del dinero, los estudiantes ven reducidos sus márgenes ele adquirir otras perspectivas respecto ele las relaciones sociales.

El sistema de control social que el poder del dinero logra articular en este modelo hace que las personas terminen convirtiéndose en engranajes del sistema mercantil y se conviertan en agentes humanos discriminatorios. Una de las maneras en que se expresa esta discriminación es en la distinción entre dos tipos de personas: las "exitosas" y las "perdedoras". las exitosas son las que consiguen acumular dinero y ascender socialmente, mientras que las perdedoras son aquellas que no consiguen acumular dinero y descienden socialmente. Pero también existen los "desechos humanos", como los ancianos, que son abandonados en departamentos solitarios y separados de las familias, o encargados a asilos de ancianos. Un segundo tipo de "desechos humanos" son los discapacitados, los que no resultan rentables ser incluidos en las instituciones educativas, o cuando son aceptados, lo son bajo la idea elela integración y nobajolaidea dela inclusión. la integración significa aplicarle la norma de la institución a los aceptados, en cambio la inclusión supone que la llegada de las personas discapacitadas reconstituye las normas y reglas de las instituciones 9 . Un tercer tipo ele "desechos humanos" son las personas de bajos recursos, para quienes el mercado asigna la educación ele peor calidad y las mínimas oportunidades ${ }^{10}$

Actualmente, en el Perú y en el resto de países de la región se está discutiendo la importancia de la educación tecnológica, es decir, la educación terciaria. Esta preocupación es completamente legítima y plausible, pues este tipo de formación es muy importante porque permite generar técnicos calificados tras dos o tres años de estudios y que pueden conseguir empleo útil con prontitud. problema reside en que el poder económico de los grupos que controlan muchas ele las universida'cles fomenta, por medio de la propaganda televisiva, que la única educación válida es la universitaria, pues ella permitirá conseguir ascenso social efectivo. la propaganda, a favor ele la educación univer sitaria, ha generado la imagen generalizada de que la educación técnica no es efectiva para el ascenso social y para conseguir un empleoadecuado.

El otro problema, en este punto, reside en que la misma educación ut versitaria se ha convertido en básicamente técnica, pues se ha expulsado la 
investigación, las humanidades y las artes y se ha concentrado en el conocimiento y la aplicación de las técnicas. Una sociedad democrática requiere de técnicos, pero una educación que se ha centrado exclusivamente en carreras técnicas, como es la tendencia actual, resulta una amenaza real para la subsístencia de la democracia.

\subsection{La educación democrática}

la educación democrática considera tanto a las artes como a las humanidades como elementos centrales, sí bien no rechazan ni a la ciencia ni a la técnica. Esta centralidad de las artes y las humanidades permite generar personas capaces de tener una actitud crítica frente a lo que se les enseña, con la capacidad de reflexión sobre lo que sucede en la sociedad y con la capacidad de imaginar sociedades diferentes. El objetivo es formar ciudadanos capaces de ser copartícipes de la conducción de las sociedades a las que pertenecen, con la capacidad de tolerar y respetar la diferencia cultural, religiosa, étnica, ele opción seJG.rnl, de visiones de vida, y capaces de incluir a personas con habilidades diferentes. Además, la educación democrática busca formar personas capaces de comprender a los demás y ser empáticos no sólo con aquellos que pertenecen a su grupo, sino con todos ciudadanos.

El cambio ele desear tener control de la sociedad, la educación de ciudadanos busca formar personas abiertas a la contingencia y a lo nuevo, capaces de vivir en un mundo social que no está a su dominio, pero en el que son capaces ele entenderse como copartícipes. De manera que la educación democrática se orienta a la formación ele ciudadanos que son capaces de reemplazar la ansiedad ele dominación y la estígmatizacíón del otro diferente porla curiosidad frentealonuevo. Elmodelo deja ele ser aquí el dominio y la coacción social, para ser reemplazado por el juego. En el juego el estudiante aprende que no todo depende ele él, sino que una parte importante de lo que suceda depende de los demás.

El juego es como el amor de pareja o el enamoramiento, en él no todo depende de una sola persona, sino también de la otra: la correspondencia, el dejarse conquistar, la apuesta por la empresa de la conquista, el respeto de los ritmos propios. En el enamoramiento y el amor ele pareja nadie es dueño de nadie, pues ambas personas son libres y es por eso que tiene su grandeza, pues en la correspondencia o en el respeto del otro brilla un reconocimiento ele la otra persona como igual, reconocimiento que colma verdaderamente nuestras expectativas. lo mismo sucede en el juego en el que se juega sin hacer trampas: los participantes se reconocen como iguales y libres, dentro de una relación que depende de tocios, tocios participan y en la cual nadie ejerce dominación. Si bien, tanto en el juego como en el amor, se plantean estrategias, ya sea para ubicarse mejor frente a los demás participantes, o para acercarse y llamar la atención a la persona que se ama, el papel ele la estrategia tiene un lugar secundario frente a la significación ele que los participantes sean libres y se encuentren sometidos unos a otros. El placer del juego se incrementa cuando los demás participantes planteen desafíos a nuestras estrategias de conducción. En el amor, el encanto desaparece cuando la persona amada se entrega ele inmediato antes de dejarse seducir. Cuando la otra persona se somete en la relación el amor desaparece y la relación se pervierte. las personas que renuncian a su libertad en el amor y pierden su autonomía dejan escapar elinterés.

la preparación para la democracia es como la preparación para el juego y el amor. En la educación democrática las personas se preparan para compartir la vida con otros en una sociedad ele personas libres e iguales, con los que deben deliberar, ponerse de acuerdo y conducir la vida ele sus sociedades de mutuo. Aquí se prepara a ciudadanos para identificarse con su país, pero que cultiven un sano nacionalismo que permita generar cíuclaclanos del mundo . la educación democrática prepara a las personas para comprender que sólo se puede tener un sano nacionalismo si se reconocen también como ciudadanos del mundo, y viceversa, sólo se es posible ser sanamente cosmopolitas sí se cultiva un sano nacionalismo $^{11}$

Además, la educación democrática fomenta la formación de una identidad compleja, lo cual amplía la capacidad de cooperación en una sociedad democrática. El fomento de una identidad compleja permite a las personas potenciar su capacidad de empatízar no sólo con los ciudadanos de su país, sino con los habitantes del planeta. Ello les permite en $t$ end erse como ciudadanos del mundo $y$ ciudadanos de su comunidad concreta, pues tiene algo que comparte, por diferentes razones, con muchas personas a lo largo del mundo. Una persona con identidad compleja tiene la capacidad de entenderse como miembro de un conjunto de comunidades diferentes, que tiene que ver con cada uno de sus focos ídentít arios . la educación democrática fomenta la identidad compleja, porque ayuda a los niños y estudiantes a mirar y a reconocer

\section{1 un1Fé}


sus diferentes aspectos identitarios sin temor y le ayuda a manejarse con la identidad compleja que lo habita sin mayor angustia. Si ciertamente es más difícil manejarse con una identidad compleja, pues ella genera ansiedades y dificultades, la educación democrática ayuda a las personas a comprender no sólo a los demás, sino a comprenderse a símismos. la educación democrática consigue esto último en dos sentidos. Primero, comprendiendo la contingencia, la animalidad que hay en nosotros y la inminencia de nuestras muertes y ayudando a manejarse frente a ellas; en segundo lugar, comprendiendo la complejidad de nuestra identidad y ayudándonos a hacernos cargo de ella. Quiero ampliar un poco este primer elemento.

la comprensión ele nuestra contingencia, de nuestra animalidad y mortalidad es muy importante para la formación de ciudadanos democráticos. la comprensión ele nuestra contingencia es importante porque permite a los ciudadanos que no podemos controlarlo ni manejarlo todo, sino que hay muchas cosas que dependen de los otros. Es por eso que las experiencias del juego y el enamoramiento son importantes. la comprensión de nuestra animalidad tiene que ver con nuestros deshechos y excrecencias. Cuando el niño aprende a ir al baño, se relaciona con una parte suya, sus heces, ele manera especial: a través de la vergüenza y la repugnancia. Pero una educación democrática le permite manejar esos sentimientos y reacciones, comprendiendo que es parte ele sus funciones vitales básicas, su animalidad. Ello le permite también mirar con comprensión y cariño sus propios errores, sus estupideces y limitaciones, como las de los demás. Respecto de la muerte, la educación democrática fomenta en las personas una sana relación con los ancianos y con los cadáveres. En cambio, discriminar a los ancianos, a causa de sus limitaciones y fragilidades, procura integrarlos como parte de la sociedad, cuidándolos y protegiéndolos. Respecto ele los cadáveres, permite comprender que es una dimensión ele nuestra condición hum...1a.

la educación democrática permite establecer una relación adecuada con la contingencia, la animalidad y la muerte. Respecto de la contingencia, permite que los estudiantes aprendan a controlar sus ansias de dominio y la angustia que genera el hecho ele no poder tener el control completo de las situaciones, sino sólo ele una parte de ella. Respecto de la animalidad y la muerte, permite manejar sanamente la vergüenza y la repugnancia, sin proyectar la repugnancia hacia otros grupos. Mientras que la educación anticlemocrática genera la distinción social entre puros e impuros, y dirige la repugnancia hacia aquellos grupos catalogados como impuros, la formación democrática permite manejar la repugnancia y dirigirla adecuadamente. Puesto que la repugnancia es una reacción que humana que tiene un componente cognitivo, puede ser dirigida a través del aprendizaje social. La escuela democrática permite hacer eso sin proyectarla hacia otros grupos, sino a través de la comprensión de los aspectos humanos que nos caracterizan. Esta comprensión es posible porque la escuela democrática fomenta el ejercicio del razonamiento, a diferencia de la escuela antidemocrática, que enfatiza el acatamiento y la sumisión a órdenes y mandatos que escapan al ejercicio delrazonamiento individual y público ${ }^{12}$.

\section{Los parámetros evaluativos}

A lo largo de lo anterior han aparecido algunas pistas para desarrollar los parámetros evaluativos de la educació.11 en cada uno de los modelos que he prest $>\mathrm{N}$ id o. En el presente trabajo me he concentrado en presentar y describir los modelos educativos, y en señalar como los modelos antidemocráticos (el aristocrático y el neoliberal) se han convertido en los dominante s en desmedro del modelo d e m o c r á t i c o . Ello resulta ser profundamente preocupante, ya que con ello se termina por promover la construcción de una sociedad en la que prevalece la discriminación dominada por el poder del dinero. Ello refuerza un sistema social perverso que es necesario examinar más a fondo a fin revertir una tendencia que se encuentra instalada en el mundo actual y poder darle su lugar al modelo democrático. En lo que sigue, presentaré sucintamente los parámetros evaluativos desarrollados por cada modelo. Dejaré, para investigaciones posteriores, el desarrollo más exhaustivo de dichos parámetros. Lo que hay que comprender es que no es posible llevar adelante esta tarea sin presentar previamente los modelos en cuestión de manera suficiente.

\subsection{Los parámetros evaluativos del modelo aristocrático}

El indicador central para la educación aristocrátic a es "verificar" si se ha interna] izado los valores domésticos y religiosos que el modelo inculca bajo la forma de "educación en valores". la educación en valores propuestos en este modelo se centra en lo que se conoce como "valores privad os", es decir, aquellos que pertenecen a una doctrina comprensiva o visión del mundo particul. $\therefore$, ya sea religiosa, filosófica o moral; pero no toma en cuenta una educación en 
valores públicos propios ele una sociedad democrática (valores como la tolerancia, el rechazo a la esclavitud, el respeto de los derechos fundamentales y los derechos humanos, entre otros $)^{13}$. Pero dicha verificación implica que la persona ha internalizado irreflexivamente dichos valores, pues en principio se presentan como principios axiomáticos sustraídos al razonamiento individual ypúblico.

Dicho indicador de verificación es problemático, porque jamás podremos saber si los individuos siguenelvalor porqueseencuentran comprometidos con él o lo hacen de manera estratégica, es decir, para adquirir una posición beneficiosa en la jerarquía dominante de la sociedad asimétrica que el modelo fomenta. Este peligro es muy real, pues al no recurrir a la reflexión y al razonamiento a fin de incorporar el valor, larelación entre los principios axiomáticos yla persona es superficial. Ello fomenta fenómenos morales contraproducentes, como la doble vida y la falsa moral.

Otro indicador evaluativo aquí resulta ser el que las personas pueden identificar lo correcto y lo incorrecto, de acuerdo a lo señalado por los axiomas valorativos, y distinguir lo "puro" de lo "impuro". Respecto de lo primero, es importante para todo sistema valorativo fomentar la capacidad de identificar lo correcto de lo incorrecto, y eso está bien; el problema reside en que esta identificación no se base en la capacidad de juicio, es decir, la capacidad reflexiva que permita acrisolar, por medio de la deliberación, los principios valorativos y poder juzgar los casos concretos de con criterio los casos particulares, sino a través del adoctrinamiento irreflexivo. Respecto de lo segundo, la distinción entre lo puro y lo impuro es altamente problemático, porque es un índice de discriminación, tal como lo he explicado arriba.

\subsection{Los parámetros evaluativos del modelo neoliberal}

El criterio evaluativo central de este modelo reside en adquirir las competencias para insertarse al mercado. Actualmente se ha impuesto en el nivel global el llamado modelo de "evaluación por competencias" 14 El término "competencia" es sumamente ambiguo. En un primer sentido significa "ser competente" para desarrollar una tarea específica. En un segundo sentido refiere a "competencias que deben adquirirse" en el proceso educativo, y en un tercer sentido, refiere a "estar habilitado para competir" en una carrera. Como veremos, el primer y el segundo sentido se encuentran conectados.

la primera acepción establece una distinción entre personas que son competentes y aquellas que no lo son, y considera que la educación haga de alguien incompetente una persona competente. Ello resulta problemático, porque no toma en cuenta las diferentes habilidades que distinguen unas personas de otras. Detrás de esta acepción se encuentra la exigencia de homogeneizar a las personas por medio de la homogenización de las habilidades en las que éstas deben ser competentes. Estas competencias promovidas por el modelo en cuestión son aquellas que hagan de la persona un funcionario eficaz para el desenvolvimiento del mercado. Como se puede observar, este primer significado se encuentra profundamente asociado al segundo significado: competencias que deben "adquirirse" en el proceso educativo.

El tercer significado, a saber, el "estar habilitado para competir" en una carrera, plantea una metáfora que resulta altamente perniciosa para la dignidad y la autoestima de las personas. la carrera como competencia supone que los educandos son competidores que se dirigen hacia una misma meta. En dicha competencia, hay quienes se encontraran relegados y quienes estarán en las primeras posiciones. Frente a la educación para "la carrera" se puede oponer la "educación como fomento de la curiosidad". Este segundo modelo, aplicado por Rabindranath Tagore en la India, se basa en ayudar a los educandos a descubrir cuáles son sus intereses más profundos estimulando la curiosidad, de manera que se entreguen por sus propias motivaciones al estudio y al trabajo en aquello que realmente los apasiona. De esta manera, los estudiantes no se encuentran insertados en una carrera que los conduce a una meta que no les interesa plenamente y que le produce frustraciones, pues ello no se conecta ni con sus intereses reales, ni con sus habilidades ${ }^{15}$.

Las competencias evaluadas en este modelo son tres: la cognitiva, la aplicativa y la valorativa. la primera señala al aprendizaje de los conocimientos en cuestión, pero no abre espacio al cuestionamiento y examen crítico de dicho conocimiento o la teoría en cuestión. La segunda competencia refiere a la capacidad de aplicar a casos específicos los conocimientos en cuestión. Finalmente, la competencia valorati va se dirige a generar un aprecio al conocimiento adquirido, pero no se debate si dicho conocimiento es apreciable o valorable en realidad. En los tres momentos del proceso de aprendizaje del sistema deeducación por competencias sedejafuera

\section{1un1Pé}


el momento de deliberación entre profesores y estudiantes respecto de lo correcto o las justificaciones que sustentan la teoría o el conocimiento enseñado. Ello es sustraído del escrutinio racional de los implicados, sino que se enfatiza la necesidad ele asumir como correctas, y las únicas correctas, las teorías queseestánenseñando.

Este fenómeno es sumamente claro en la enseñanza que se imparte en muchas escuelas ele economía en el Perú: existe una corriente económica dominante y ésta es enseñada como sí fuese una verdad absoluta, incuestionable y valorable por sí, de manera que los estudiantes deben ele aprender a aplicar a casos concretos. Lo cierto es que la teoría dominante en las escuelas económicas, basada en el modelo clásico articulado por Adam Smith y Milton Freeclman, está siendo cuestionado por nuevas corrientes económicas y economistas alternativos con argumentos sólidos, pero dichos cuestionamientos no son estudiados en muchas universidades. Otro ejemplo es la enseñanza de la corriente ele pensamiento jurídico del Análisis Económico del Derecho como si fuese la concepción del derecho correcta, la cual es asumida sin cuestionamiento.

Finalmente, el modelo neoliberal de evaluación por competencia exige que las evaluaciones tengan una estructura que expulse la redacción, por parte de los alumnos, de textos en los que discutan y critiquen los modelos aprendidos. El enfoque se centra, más bien en preguntas tipo "marcar la alternativa correcta" (opción múltiple), "establecer las relaciones correctas entre enunciados propuestos" o "escribir la definición correcta de conceptos". Todas estas estrategias premian la memoria, pero no el razonamiento y menos el razonamiento crítico.

Como es posible observar, e] modelo ele evaluación neoliberal existe una poderosa tendencia dogmática que consagra como verdaderas un conjunto de conocimientos, doctrinas y teorías. En este sentido se asocia con eJ. modelo aristocrático. En realidad, ambos modelo s han establecido una alianza estratégica para encasillar a la sociedad en un esquema de relaciones que permite mantener los privilegios de las élites, utilizando la educación como una herramienta poderosa porque a través ele ella pueden modelar el espíritu de los ciudadanos. En dicha alianza, los aristócratas consiguen que el conservadurismo cultural se preserve, ele tal manera que las culturas subordinadas se mantengan en su sitio sin atentar al sistema con sus reivindicaciones y sus exigencias de reconocimiento. Por su parte, el modelo ncoliberal consigue hacer que el modelo económico no se altere a fin de que sus intereses económicos no se vean amenazados. Esta alianza se ve con transparencia en muchas instituciones educativas regentadas por movimientos conservadores radicales, donde se procura mantener las "buenas costumbres" y se fomenta el espíritu de lucro. Los agentes y las instituciones participantes en dicha alianza estratégica encuentran en el modelo democrático un peligro, es por ello que utilizan todos sus recursos para mantenerlo a raya, pues se trata de un modelo cuestionador.

\subsection{Los parámetros evaluativos del modelo democrático}

El modelo democrático fomenta el autoexamen y la deliberación compartida ele los conocimientos, de manera que fomenta el pensamiento crítico. Los sectores conservadores han cuestionado el fomento del pensamiento crítico utilizando un conjunto de estrategias erráticas. Por ejemplo, se ha señalado que la crítica no se puede radicalizar, porque de lo contrario se convierte en un ejercicio vacío, sin ningún contenido. Esta crítica se asocia a la crítica como un ejercicio simplemente formal ${ }^{16}$. En realidad, tales críticas al pensamiento crítico caen en dos problemas centrales. En primer lugar, realizan algo que supuestamente cuestionan: procuran dar argumentos para cuestionar una teoría o un modelo de educación, cayendo en lo que se conoce corno una contradicción performativa. En segundo lugar, sus argumentos resultan ser falaces, pues siempre terminan cayendo en la llamada falacia "recurso a la autoridad", que señala que si alguna autoridad o la tradición han señalado algo, entonces ello resulta ser incontestable.

El modelo democrático coloca como indicadores evaluativos la capacidad ele razonar y ele imaginar. El razonamiento tiene corno finalidad el sopesar la justificación ele los conocimientos ofrecidos en el proceso de aprendizaje, ciertamente ele manera progresiva, de acuerdo a la madurez que vaya adquiriendo el educando. La imaginación tiene dos objetivos centrales, en primer lugar, fomenta la capacidad ele pensar conocimientos y modelos alternativos; en segundo lugar, fomenta la capacidad ele colocarse en el lugar del otro, de manera que fomenta la empatía. Para ello es indispensable la inclusión de las artes y las humanidades. Las herramientas fundamentales utilizadas por este modelo son la discusión en clase, el intercambio ele ideas, razones y argumentos. En este proceso de discusión los estu,.iantes traen al debate sus experiencias de vida y sus observaciones 
particulares, es por ello que fomenta el autoexamen y la observación crítica del mundo que lo circunda. En el debate mismo los educandos adquieren el hábito de revisar sus prejuicios respecto de formas de vivir diferentes a la suya y de grupos étnicos y sociales diferentes. la discusión en clase de diversos temas es un mecanismo efectivo para desaprender prejuicios ${ }^{17}$. Otra herramienta :importante la constituye los trabajos de investigación y búsqueda que fomentan la curiosidad delos estudiantes .

Pero el indicador del aprendizaje es la capacidad que tienen los estudiantes de asociarse para hacer cosas con sus propias manos y para poder gestar en común propuestas y la capacidad de llevarlas adelante. Ello los prepara para el compromiso con la democracia. El modelo democrático tiene como meta formar ciudadanos empáticos y comprometidos, y esa formación se logra en un ejercicio constante de las prácticas constantes de actitudes democráticas fundamentales en la escuela y en la universidad. Estas actitudes son la tolerancia, la apertura a la deliberación, la participación en empresas compartidas. Todas estos, junto con el rechazo a la discriminación, el reconocimiento de los derechos fundamentales, etc., constituyen los llamados valores públicos. Aquí hay una diferencia central entre la educación aristocrática y la educación democrática. la primera apunta al fomento de valores privados, mientras que la segunda apunta a los valores públicos. Mientras que los valores privados se imponen como axiomáticos, los valores públicos son aceptados voluntariamente por los ciudadanos tras un proceso de deliberación público que permite definirlos y redefinidos a través de la argumentación y la discusión racional.

\section{Conclusiones: la educación como amenaza a la democracia}

Tanta la educación aristocrática como la neoliberal se convierten en amenazas a la democracia debido a su talante autoritario. la proliferación de instituciones educativas que no se encuentran auténticamente comprometidas con la democracia se conviertan en espacios de formación de personas que son incapaces de cultivar la empatía, derechazar la discriminación y que son incapaces de manejar adecuadamente supropiafinitudy la relación conlos aspectos repugnantes de sí mismos. El predominio que los modelos antidemocráticos están tomando en el Perú y en el mundo resulta ser algo preocupante frente a lo cual hemos de llevar medidas concretas, personales e institucionales que transformen la política pública educativa, la malla curricular y los métodos evaluativos. Pero, sobre todo, es necesario transformar las relaciones que establecen en las aulas, y las relaciones entre los docentes y las autoridades de las instituciones educativas, para que dejen de ser autoritarias y se transformen en democráticas. De otro modo, el futuro de las sociedades contemporáneasserá sombrío.

Si la evaluación de la educación tiene una relación directa con el modelo de sociedad para la que se está formando a las personas, es necesario tener en cuenta que la evaluación tiene como punto de vista firme la siguiente pregunta: lqué tan preparados para vivir en una sociedad democrática y qué tan comprometidos se encuentran las personas que las instituciones educativas están formando? Aquí aparecen dos niveles de evaluación que deben estar en concordancia. El primer nivel es el de la evaluación de las propias instituciones educativas; mientras que el segundo nivel de evaluación tiene el ele los educandos al interior de las mismas instituciones educativas .

Normalmente se ha discutido en torno al segundo nivel evaluativo, debido a que se ha presupuesto que hay un acuerdo respecto del primer nivel, pero sucede que dicha creencia se ha mostrado falsa. El primer nivel de evaluación dela educación exige que las instituciones educativas sean democráticas en dos sentidos: han de tener una organizaci democrática, en el sentido de que la relación entre los docentes, alumnos y autoridades ha de ser democrática. En las escuelas, debe desterrarse los tratos autoritarios y en las universidades, además, las autoridades deben ser elegidas democráticamente. Pero además, las instituciones han de ser democráticas en un segundo sentido, han de asumir la tarea de formar ciudadanos, de modo que deben integrar a las humanidades y las artes como elementos fundamentales para formar personas para la democracia.

En el segundo nivel de la evaluación lo que se toma como centro es la manera en que los estudiantes van adquiriendo los hábitos para desarrollarse como ciudadanos: la capacidad de participar en los intereses comunes, y no sólo replegados a sus intereses particulares, la apertura al diálogo y la capacidad de argumentar, expresando sus argumentos con claridad y precisión, y estando dispuestos a reconocer los mejores argumentos. Además, los educandos han de ir preparándose para ser tolerantes con las personas que tienen concepciones religiosas y de la vida distintas. los educandos han de comprometerse con los derechos

\section{1 un1Pé}


fundamentales, los derechos humanos y la democracia y se encuentren dispuestos a rechazar las violaciones de los derechos y a descalificar a los regímenes políticos antidemocráticos.

Si no tenemos claridad en torno a estos dos niveles de la evaluación de la educación no será posible que orientemos adecuadamente nuestro pensamiento al respecto y nuestra actividad educativa será tergiversada por la influencia de los radicalismos y por la influencia del poder del dinero. Si queremos preservar la libertad y la igualdad democrática es necesario que las instituciones educativas tengan en claro ambos niveles de evaluación y han de combatir los intentos de las fuerzas antidemocráticas que están ganando cada vez más terreno en Perú y en las sociedades contemporáneas. Al igual que el Sócrates de La República, puedo decir que lo que estamos tratando está lejos de ser algo banal, pues tiene que ver con la manera en que hemos de conducir nuestras vidas en tanto ciudadanos.

\section{NOTAS}

(1) C/ Dewey , John (1995). Democra cia y educación: 1111a introdl1ccioli a._la filosofia de la educación. Madrid: Morata; y Nussbaum, Mattl:ia (2010). Sin fines de /11cro. Porqué la democracia necesitdr as humanidades. Buenos Aires: Katz.

(2) C.f. Dewey, John (1995). Ob. Cit.

(3) Sobre el particular, $C f$ Tugendhat, En s t (199 7). lecciones de ética. Barcelona: Gedisa.

(4) ei: Mackie, John (2000). Ética: la investiga ción de lo bueno y lo mal. Barcelona: Ged is a; Nagel, Thomas (1996). Una visión de ningún fuga México: FCE; yTugendhat, Enst. Ob. Cit.

(5) Al respecto de la complejidad de la identidad, Cf Maalouf, Amin (2002). Identidades asesinas. Madrid: Alianza Editorial; y Sen, Amartya (2007). Identidad y violencia. la ilusión del destino. Buenos Aires: Katz.

(6) Durante el gobierno de Alejandro Toledo se realizaron intentos de incluir ese aspecto de la historia en los textos esco lares, pero al asumir el mando Alan García todo proyecto dirigido a ese propósito fue interrumpido. Ciertamente, no es noticia para nadie el que Alan García estableció una alianza política tanto con el fujimorismo como con los sectores más conservadores de la Iglesia Católica. Muestra de esa alianza fue la designación de Cecilia Bákula como directora del INC, cuya gestió1 ignifícó un gran retroceso en la promoción de las artes y la cultura de parte del Estado, como el maltrato a muchos artistas, como en el caso de los maltratos sistemáticos a los miembros del Ballet Nacional y a los de la Orquesta Sinfónica.

(7) Respecto de la predilección de la educación antidemocrática por la ciencia, la técnica y la historia Cf Nussbaum, Martha. Ob. Cit.
(9) Carvallo, Constantino (2011 ). Dond e habite la moral. Reflexiones sobre.fi!osofiayeducación. Lima: Aguilar. p. 182.

\{10) Por ejemplo, en Chile sucede que los qu int il es más bajos reciben peor educación tanto de parte de la escuela púhlica como de la privada , mient ras que en el Perú sucede que en esos sectores la educación pública es superior a la privada.

(11) C/ Appiah , Anth ony (2007). Cosl11opo!itis1110: 1111a ética para 1111 mil11do de extraiíos. Buenos Aires: Kat z.

(12) Con relación a esto Cf Nussbau rn, Martha (2006). El ocultamiento de/o humano. Repugnancia, vergüenza y ley Bue nos Aires: Katz.

(13) Para ver la diferencia entre los valores públicos y los valores domésticos Cf Rawls, John (1996). lihera/ismo político. México: FCE. Conferencias I y 4.

(14) Uno de los rnodelos rnás extendidos en los paíse s latinoarnericanos es el llamado "Protoco lo de Boloña".

(15) Resp ecto de 1 trabajo educativo de Tagore en la India, Cf Sen, Amartya (2007). La India contemporánea. Entre la modernidad y la tradi ción. Madr id:Gedisa.

(16) Un ejemplo de ello lo constituye la crítica conservad ora al método his tóric o- crítico en la exégesis bíblica.

( 1 7) C/ Gutmann, Amy (2001 ). la educación democrática. Una teoría política de la educación. Barcelona: Paidós. También véase Stem-La Rosa, Cary l y 1-lofheimer Bettmann, Ellen (2000). The A11tiDe/allatioll I e ague's Hale Hurts. How Clii lden Leam and Unleam Prejudice. New York: Anti - Defamation Leag ue's .

(8) C.f Nug e nt, Guillenno. Ob. Cit.

Mg. Alessandro Caviglia Marconi

Magíster en Filosofía por la Pontificia Universidad Católica del Perú. Profesor del Departamento de Filosofía y Teología de la Universidad Femenina del Sagrado Corazón, profesor de la Carrera de Filosofía de la Universidad Antonio Ruiz de Montoya, profesor del Departamento de Humanidades de la Univ t., si dad Ricardo Palma y profesor de la Facultad de Derecho de la Universidad de San Martín de Parres. 